\title{
Idiopathic pulmonary fibrosis: time for greater expectations?
}

\author{
Wim Wuyts ${ }^{1}$ and Vincent Cottin ${ }^{2}{ }^{2,3}$
}

Affiliations: 'University Hospitals Leuven, Unit for Interstitial Lung Diseases, Dept of Pulmonary Medicine, Leuven, Belgium. ${ }^{2}$ Hospices Civils de Lyon, Dept of Respiratory Medicine, National Reference Center for Rare Pulmonary Diseases, Lyon, France. ${ }^{3}$ Claude Bernard Lyon 1 University, University of Lyon, INRA, Lyon, France.

Correspondence: Vincent Cottin, Hopital L. Pradel - Service de Pneumologie, 28 Avenue Doyen Lepine, Lyon 69677, France. E-mail: vincent.cottindachu-lyon.fr

@ERSpublications

Based on two studies published in the ERJ, maybe the time has come to raise the bar and target larger goals for IPF treatment when designing new treatment approaches, goals that might better fit patients' expectations http://ow.ly/MsJR30kZtPf

Cite this article as: Wuyts W, Cottin V. Idiopathic pulmonary fibrosis: time for greater expectations? Eur Respir J 2018; 52: 1801312 [https://doi.org/10.1183/13993003.01312-2018].

Idiopathic pulmonary fibrosis (IPF) is a relentless fibrotic disorder, ultimately leading to respiratory insufficiency and death. It is characterised by a progressive and irreversible damage of lung tissue, leading to progressive dyspnoea, decrease in pulmonary function and worsening features of fibrosis on imaging. Although initially considered an inflammatory disorder, after many years of research, the paradigm shifted to that of a predominantly fibrotic disorder [1], which was an important milestone in the history of IPF [2]. As a result, drug development later focused on agents primarily targeting the progression of fibrogenesis, i.e. fibroblast proliferation and secretion of extracellular matrix. Pirfenidone and nintedanib were the first drugs to demonstrate efficacy as antifibrotic agents [3-6], and were rapidly approved worldwide.

As mortality did not seem practically feasible as a primary endpoint, trials in IPF have been conducted using endpoints known to be associated with mortality during the subsequent year of follow-up [7-9]. Hence, forced vital capacity (FVC) decline has been considered a surrogate endpoint of mortality and has been used as the primary endpoint in most IPF trials, but with some variation in methods used to calculate FVC decline or the thresholds used. Overall, the data from pivotal trials [3-6] have shown that antifibrotic agents slow the rate of decline in FVC over time in comparison with placebo. However, the natural history of IPF is highly variable and predicting the prognosis in an individual patient is challenging. Some patients have a rapid decline, others progress more gradually or experience periods of apparent stability, with further episodes of abrupt decline or acute exacerbations $[10,11]$.

Due to the chronic, irreversible, relentless progression of IPF causing irreversible damage to the lung, it is generally considered that the goal of therapy can at best be to stabilise the disease course. In trials, power and sample size calculations are based on the assumption that the drug under development will hopefully reduce disease progression by a third to a half of its natural potential for worsening. But are we so sure that this is the best we can do for our patients? The fact that some patients might experience pulmonary function improvement after initiation of treatment has so far only been reported in real-life experience series of patients who had received pirfenidone [12].

In this issue of the European Respiratory Journal (ERJ), FLAHERTY et al. [13] report on a post hoc analysis of the distribution of changes in FVC in the INPULSIS trials [6]. They show that $24.8 \%$ of patients 
randomised to receive nintedanib and $9.0 \%$ of patients receiving placebo had a numerical increase or no decline in FVC over the duration of the trial (52 weeks). The median (interquartile range) improvement was $76.5 \mathrm{~mL}(31-152 \mathrm{~mL})$ in the nintedanib group and $57.5 \mathrm{~mL}(31-103 \mathrm{~mL})$ in the placebo group, among subjects in whom FVC increased.

These results are, of course, tantalising. Patients receiving nintedanib were 2.75 times more likely to experience an improvement in FVC at 1 year. However, such results are mainly helpful in challenging the current, and possibly pessimistic, dogma that treatment can only slow down or, at best, stabilise FVC. There are, however, a number of issues and unknowns to consider, and it certainly cannot be concluded that genuine improvement of this dismal condition can yet be obtained with drug therapy.

First of all, an apparent increase in FVC was seen in 9\% of patients receiving placebo. Knowing the natural course of IPF when left untreated, such a finding may simply reflect measurement variability in FVC or transient stability of IPF rather than true reversal in the course of disease. Indeed, "improvement" was defined by any numerical increase in the annual rate of change in FVC calculated by a random coefficient regression model. Variability in FVC measurement is well known; however, significant efforts were made in the INPULSIS trials to reduce it [6]. The minimal clinically important difference of FVC was previously estimated to be between $2 \%$ and $6 \%$ in IPF [14]. Participating in a clinical trial with rigorous follow-up and strict procedures in place might lead per se to a better outcome than in the general patient population; however, this applies to both groups in the trial. Eligibility criteria may also have contributed to a more favourable result, as only patients with mild to moderate severity of disease and a strict limitation of comorbidities were enrolled in the trials, differing from daily clinical practice, but again this would not explain the difference seen between treatment groups.

More important reasons to be cautious in evaluating these results need be mentioned. Clinicians treating patients with IPF do occasionally observe disease stabilisation with antifibrotic therapy; however, genuine long-term improvement does not occur in patients with a secure diagnosis of IPF. The majority of individuals with IPF continue to die prematurely from their disease. When monitoring IPF progression, parameters other than FVC do matter, such as symptoms or change in thoracic imaging, and an unfavourable outcome may not always be preceded by FVC decline on an individual basis [15]. FVC decline is not linear and past decline in FVC does not predict future decline in FVC [16]. The clinical significance of a "stable or improved FVC" is not well known, although in a study conducted by investigators from the Food and Drug Administration, patients experiencing a decline in FVC of $\geqslant 5 \%$ had increased risk of death compared to those who did not, suggesting that even marginal decline in FVC may be meaningful [17] and that the absence of any decline may be favourable. The long-term outcome of the patient group with stable or improved FVC is unknown with regards to hospitalisations, acute exacerbations, radiographic worsening and eventual survival. Notably, the authors could not identify any baseline characteristics that would predict an improvement in FVC.

Altogether, it seems currently improbable that sustained and clinically significant improvement in lung function may be obtained with either nintedanib or pirfenidone alone in patients with IPF. More optimism may be reasonable in fibrotic lung diseases other than IPF, which often are less severe and not seldom less progressive, and the results of trials evaluating the tolerability and efficacy of antifibrotic drugs in these conditions are eagerly awaited (ClinicalTrials.gov NCT03099187) [18-20].

Further improvement in IPF might come from combination therapy [21], similarly to what already has been achieved in other chronic diseases including asthma, chronic obstructive pulmonary disease and pulmonary arterial hypertension. Despite much hope that combination therapy may improve treatment efficacy, careful evaluation is needed before it can be used in the clinic. Past experience has clearly demonstrated that combining drugs could, in theory, potentiate tolerance or safety issues [22, 23] and that the benefit of combining drugs may not always be additive.

In this context, FLAHERTy et al. [24] publish, in this issue of the ERJ, the results of a phase II trial combining nintedanib with pirfenidone in patients with IPF. In this 24 -week, single-arm, open-label, phase IV study, patients who had already received pirfenidone for $\geqslant 16$ weeks for IPF and who tolerated a stable dose of 1602-2403 mg per day without experiencing any moderate or severe adverse reactions related to the drug were initiated with nintedanib added to pirfenidone. Out of 89 patients, $69(78 \%)$ completed 24 weeks of combination treatment. Despite this high proportion considered to tolerate the combined treatment, it should be emphasised that almost all patients experienced treatment-emergent adverse events, including half of them (49\%) who had diarrhoea and $7 \%$ who experienced treatment-related hepatic adverse events, which were generally considered to be related to nintedanib due to the known tolerability profile of this drug. Indeed, investigators were asked to which of the two drugs they attributed the adverse events, although this statement seems to be very challenging in patients receiving a combined treatment. In any combination therapy when drugs are initiated sequentially, only 
selected patients who tolerate the "first" drug well can be eligible to receive the "second" drug, which by default, but possibly spuriously, would be accused of any new adverse event.

Results of this important study are consistent with those of the recent 12-week INJOURNEY trial [25], in which pirfenidone or placebo were randomised and added to nintedanib treatment, both drugs being used at the usual dose. Both studies found the combination therapy to be tolerable in a majority of preselected patients that tolerate one of these two drugs, and no unanticipated adverse event emerged. The fact, however, that around $20-25 \%$ of patients did not tolerate the combination despite dose adjustment strategies already in place suggests that the long-term feasibility of such dual therapy might be challenging in a proportion of patients. This is, however, an avenue that is worth pursuing, and the efficacy of the combination now needs to be carefully assessed. In the single-arm exploratory study by FLAHERTY et al. [24], all patients received the combination therapy, a design which makes it impossible to assess the efficacy of combination versus monotherapy; however, it is encouraging that there was little decline in lung function during the duration of the trial (combination therapy) with a trend towards less decline in FVC as compared to the pre-trial period (pirfenidone monotherapy). In an exploratory analysis of the INJOURNEY trial [25], the mean \pm SE changes from baseline in FVC at week 12 were $-13.3 \pm 17.4 \mathrm{~mL}$ and $-40.9 \pm 31.4 \mathrm{~mL}$ in patients treated with nintedanib with add-on pirfenidone and nintedanib alone, respectively. In another study not yet published, no significant interaction was found between nintedanib and pirfenidone. It should be stressed that these data are exploratory, and important hurdles have to be passed before combination therapy can be considered an efficient option for patients with IPF.

Together, these studies make important contributions to the field. A combined treatment with nintedanib and pirfenidone may be feasible. It is also now conceivable that long-term stabilisation of disease may be obtained with antifibrotic therapy and that a proportion of patients may experience some improvement in lung function. Maybe the time has come to raise the bar and target larger goals for IPF treatment when designing new treatment approaches, goals that might better fit patients' expectations than our current options of slowing the rate of disease worsening.

Conflict of interest: W. Wuyts reports that he is on the speakers' bureau for both Roche and Boehringer Ingelheim. His institution has received grants from both Boehringer Ingelheim and Roche. V. Cottin reports receiving consultancy and lecture fees and support for travel to medical conferences from Actelion; a grant to his institution, personal fees for the development of educational presentations, consultancy and lecturing, and support for travel to medical conferences from Boehringer Ingelheim; consultancy fees from Bayer/MSD and GSK; personal fees for membership of a adjudication committee from Gilead; consultancy and lecture fees from Novartis; a grant to his institution, consultancy and lecture fees, and support for travel to medical conferences from Roche; a grant for coordination of a trial from Sanofi; personal fees for chairing a data and safety monitoring board (DSMB) from Promedior; personal fees for serving on a DSMB from Celgene; and consultancy fees from Galapagos, all outside the submitted work.

\section{References}

1 Selman M, King TE, Pardo A, et al. Idiopathic pulmonary fibrosis: prevailing and evolving hypotheses about its pathogenesis and implications for therapy. Ann Intern Med 2001; 134: 136-151.

2 Wells AU, Brown KK, Flaherty KR, et al. What's in a name? That which we call IPF, by any other name would act the same. Eur Respir J 2018; 51: 1800692.

3 Noble PW, Albera C, Bradford WZ, et al. Pirfenidone in patients with idiopathic pulmonary fibrosis (CAPACITY): two randomised trials. Lancet 2011; 377: 1760-1769.

4 King TE Jr, Bradford WZ, Castro-Bernardini S, et al. phase 3 trial of pirfenidone in patients with idiopathic pulmonary fibrosis. N Engl J Med 2014; 370: 2083-2092.

5 Richeldi L, Costabel U, Selman M, et al. Efficacy of a tyrosine kinase inhibitor in idiopathic pulmonary fibrosis. N Engl J Med 2011; 365: 1079-1087.

6 Richeldi L, du Bois RM, Raghu G, et al. Efficacy and safety of nintedanib in idiopathic pulmonary fibrosis. $N$ Engl J Med 2014; 370: 2071-2082.

7 Raghu G, Collard HR, Egan JJ, et al. An official ATS/ERS/JRS/ALAT statement: idiopathic pulmonary fibrosis: evidence-based guidelines for diagnosis and management. Am J Respir Crit Care Med 2011; 183: 788-824.

8 Flaherty KR, Mumford JA, Murray S, et al. Prognostic implications of physiologic and radiographic changes in idiopathic interstitial pneumonia. Am J Respir Crit Care Med 2003; 168: 543-548.

9 du Bois RM, Weycker D, Albera C, et al. Six-minute-walk test in idiopathic pulmonary fibrosis: test validation and minimal clinically important difference. Am J Respir Crit Care Med 2011; 183: 1231-1237.

10 Ley B, Collard HR, King TE Jr. Clinical course and prediction of survival in idiopathic pulmonary fibrosis. Am J Respir Crit Care Med 2011; 183: 431-440.

11 Collard HR, Ryerson CJ, Corte TJ, et al. Acute exacerbation of idiopathic pulmonary fibrosis. An International Working Group Report. Am J Respir Crit Care Med 2016; 194: 265-275.

12 Leceuvre K, De Dycker E, Moerman T, et al. Efficacy of pirfenidone in idiopathic pulmonary fibrosis: a single center experience. Eur Respir J 2016; 48: Suppl. 60, PA2088.

13 Flaherty KR, Kolb M, Vancheri C, et al. Stability or improvement in forced vital capacity with nintedanib in patients with idiopathic pulmonary fibrosis. Eur Respir J 2018; 52: 1702593.

14 du Bois RM, Weycker D, Albera C, et al. Forced vital capacity in patients with idiopathic pulmonary fibrosis: test properties and minimal clinically important difference. Am J Respir Crit Care Med 2011; 184: 1382-1389. 
15 Martinez FJ, Safrin S, Weycker D, et al. The clinical course of patients with idiopathic pulmonary fibrosis. Ann Intern Med 2005; 142: 963-967.

16 Schmidt SL, Tayob N, Han MK, et al. Predicting pulmonary fibrosis disease course from past trends in pulmonary function. Chest 2014; 145: 579-585.

17 Richeldi L, Ryerson CJ, Lee JS, et al. Relative versus absolute change in forced vital capacity in idiopathic pulmonary fibrosis. Thorax 2012; 67: 407-411.

18 Flaherty KR, Brown KK, Wells AU, et al. Design of the PF-ILD trial: a double-blind, randomised, placebo-controlled phase III trial of nintedanib in patients with progressive fibrosing interstitial lung disease. BMJ Open Respir Res 2017; 4: e000212.

19 Distler O, Brown KK, Distler JHW, et al. Design of a randomised, placebo-controlled clinical trial of nintedanib in patients with systemic sclerosis-associated interstitial lung disease (SENSCISTM). Clin Exp Rheumatol 2017; 35: Suppl. 106, S75-S81.

20 Behr J, Neuser P, Prasse A, et al. xploring efficacy and safety of oral Pirfenidone for progressive, non-IPF lung fibrosis (RELIEF) - a randomized, double-blind, placebo-controlled, parallel group, multi-center, phase II trial. BMC Pulm Med 2017; 17: 122.

21 Wuyts WA, Antoniou KM, Borensztajn K, et al. Combination therapy: the future of IPF management? Lancet Respir Med 2014; 2: 933-942.

22 Raghu G, Anstrom KJ, King TE Jr, et al. Prednisone, azathioprine, and N-acetylcysteine for pulmonary fibrosis. N Engl J Med 2012; 366: 1968-1977.

23 Behr J, Bendstrup E, Crestani B, et al. Safety and tolerability of acetylcysteine and pirfenidone combination therapy in idiopathic pulmonary fibrosis: a randomised, double-blind, placebo-controlled, phase 2 trial. Lancet Respir Med 2016; 4: 445-453.

24 Flaherty KR, Fell CD, Huggins JT, et al. Safety of nintedanib added to pirfenidone treatment for idiopathic pulmonary fibrosis. Eur Respir J 2018; 52: 1800230.

25 Vancheri C, Kreuter M, Richeldi L, et al. Nintedanib with add-on pirfenidone in idiopathic pulmonary fibrosis. Results of the INJOURNEY trial. Am J Respir Crit Care Med 2018; 197: 356-363. 